\title{
A Fractal-Based Approach to Network Characterization Applied to Texture Analysis
}

\author{
Lucas C. Ribas ${ }^{1}$, Antoine Manzanera ${ }^{3}$, and Odemir Bruno ${ }^{2}$ \\ 1 Institute of Mathematics and Computer Science, University of São Paulo - USP, \\ Avenida Trabalhador são-carlense, 400, 13566-590, São Carlos, SP, Brazil \\ lucasribas@usp.br \\ 2 São Carlos Institute of Physics, University of São Paulo - USP, PO Box 369, \\ 13560-970, São Carlos, SP, Brazil \\ bruno@ifsc.usp.br \\ 3 U2IS, ENSTA Paris, Institut Polytechnique de Paris, 828 Boulevard des \\ Maréchaux, 91120 Palaiseau, France \\ antoine.manzanera@ensta-paristech.fr
}

\begin{abstract}
This work proposes a new method for texture analysis that combines fractal descriptors and complex network modeling. At first, the texture image is modeled as a network. Then, the network is converted into a surface where the Cartesian coordinates and the vertex degree is mapped into a $3 \mathrm{D}$ point in the surface. Then, we calculate a description vector of this surface using a method inspired by the BouligandMinkowski technique for estimating the fractal dimension of a surface. Specifically, the descriptor corresponds to the evolution of the volume occupied by the dilated surface, when the radius of the spherical structuring element increases. The feature vector is given by the concatenation of the volumes of the dilated surface for different radius values. Our proposal is an enhancement of the classic complex networks descriptors, where only the statistical information was considered. Our method was validated on four texture datasets and the results reveal that our method leads to highly discriminative textural features.
\end{abstract}

Keywords: Complex Networks · Fractal Dimension · Texture analysis.

\section{Introduction}

Texture is a visual pattern related to the surface of a material or an object and it is considered as a key feature to image interpretation. Texture classification is used in many fields such as material science [43], industrial inspection [29], geology [42], etc. There are many classical texture characterization methods in the literature that can be divided into four different categories: statistical-based (e.g., gray-level co-occurrence matrices (GLCM) [22] and local binary patterns (LBP) [32]), spectral methods (e.g. Gabor filters [24] and wavelet transform [41]), structural methods (e.g. morphological decomposition [27]) and model-based methods (e.g. Fractal models [6,5,37], complex networks [36] and stochastic models [34]) 
Currently, methods based on complex networks theory have attracted significant attention of the computer vision community, due to the promising results and their capacity to represent the relationships among structural properties of texture $[15,38,1]$. The complex network techniques represent the texture image as a network and extract measures capable of characterizing precisely the structural patterns of texture modeled. In this sense, the network modeling is an important step whose aim is to represent different aspects like spatial and pixel intensity distribution, texture irregularities, auto-similarity scales, etc. [15]. Now a challenging problem is to find a relevant set of measures to extract from the network to characterize it. Previous works $[7,10]$ use simple metrics based on degree distribution, however, it is noticeable that more complex measures are needed for a finer characterization [15, 12].

To this end, we propose in this paper a new approach for texture analysis that uses fractal descriptors as measures for complex network characterization. The proposal is to use the fractal measures to characterize the network topology instead of using network statistical measures. To achieve this, firstly, the texture image is modeled as a network and this network is converted to a surface. Then, we calculate a feature vector using a method inspired by the BouligandMinkowski technique to estimate the fractal dimension of the surface that maps a network. The proposed signature is composed of the influence volume computed of this surface for different radius values. We verify the performance of the proposed approach in the classification of four texture image datasets and the results are compared to other literature methods.

The paper is organized as follows. In Section 2, we describe the proposed approach in detail. Section 3 reports the experimental setup. The results and discussion are presented in Section 4, followed by the conclusion in Section 5 .

\section{Proposed Method}

\subsection{Modeling texture as network}

According to Backes et al [7] a texture image $I$ can be modeled as a network $G=(V, E)$ considering each pixel as a vertex. Each pixel $i \in V$ is characterized by its Cartesian coordinates $x_{i}$ and $y_{i}$, and an integer value $I(i) \in[0,255]$ which represents its gray-level. Two vertices (pixels) $i$ and $j$ are connected by a nondirected edge $e_{i j} \in E$ if the Euclidean distance between them is smaller or equal than a given value $d$, according to:

$$
e_{i j} \in E \Longleftrightarrow \sqrt{\left(x_{i}-x_{j}\right)^{2}+\left(y_{i}-y_{j}\right)^{2}} \leq d
$$

For each edge a weight $w\left(e_{i j}\right)$ is defined by a normalized combination of the Euclidean distance and the difference of intensities between two pixels:

$$
w\left(e_{i j}\right)=\frac{\left(x_{i}-x_{j}\right)^{2}+\left(y_{i}-y_{j}\right)^{2}+d^{2} \frac{|I(i)-I(j)|}{255}}{2 d^{2}}
$$


This weight function includes information about the pixel surroundings and the normalization of the difference of pixel intensity in the interval $\left[0, d^{2}\right]$ aims to balance the relative importance between geometric and color information in the texture representation [7]. Each network vertex $i$ has a degree $k_{i}$, which is the number of edges connected to $i$. Note that until now the network vertices all have the same degree. Thus, it is necessary to apply a transformation in order to highlight texture properties. This can be done by applying a threshold $t$ over the network, removing all edges whose weight is higher than a given value $t$ and obtaining a new set of edges $E_{t}$. In this way, a set of thresholds $T, t \in T$ can be applied over the network in order to study the behavior of its properties along the successive transformations.

\subsection{Fractal Dimension of Network Degrees}

A way to describe how irregular is an object is to use the Fractal dimension, which can be estimated in various ways [17]. In this paper, we compute the fractal descriptors of the complex network using a method inspired by the BouligandMinkowski method, which is one of the most accurate approach to estimate the fractal dimension [4,9]. This method estimates the fractal dimension based on the size of the influence area $|S(r)|$ generated by the dilation of surface $S \subset \mathbb{R}^{3}$ using a radius $r$ [17]. The Bouligand-Minkowski fractal dimension $D$ of a surface $S$ varying the radius $r$ is defined as:

$$
D(S)=3-\lim _{r \rightarrow 0} \frac{\log V_{S}(r)}{\log r},
$$

where $V_{S}(r)$ is the influence volume achieved with the dilation process of all points $s$ of the surface $S$ using a sphere of radius $r[17,4] . V_{S}(r)$ can be calculated by the Euclidean Distance Transform (EDT) in order to achieve a fast algorithm $[4,13]$. The distance transform $D T$ is the function defined as:

$$
D T(x, y, k)=\min _{\left(x^{\prime}, y^{\prime}, k^{\prime}\right) \in S} d\left((x, y, k),\left(x^{\prime}, y^{\prime}, k^{\prime}\right)\right),
$$

for all points $(x, y, k)$ from the cubic grid $\mathbb{N}^{3}[4,39]$. This way, the set of possible radii (distances) $R$ is

$$
R=\left\{r: r=\sqrt{x^{2}+y^{2}+k^{2}} ;(x, y, k) \in \mathbb{N}^{3}\right\},
$$

with the values $r$ sorted increasingly to create the set of possible radii $R=$ $\left\{0,1, \sqrt{2}, \sqrt{3}, 2 \sqrt{2}, \ldots, r_{\max }\right\}$ [39]. In this approach, the influence volume is computed by $V_{S}(r)=\#\{(x, y, k): D T(x, y, k)=r\}$ [39].

In the proposed method, we apply the fractal dimension theory to characterize the topology of a network that models a texture image. To achieve this, a network modeling texture can be easily mapped onto a $3 \mathrm{D}$ surface $S \subset \mathbb{R}^{3}$, by converting the Cartesian coordinates $\left(x_{i}, y_{i}\right)$ and the degree $k_{i}$ of each vertex $i$ into a 3D point $s_{i}=\left(x_{i}, y_{i}, k_{i}\right)$. In this way, we can characterize the network topology relating the degree and the vertex/pixel position in the image. Figure 
1(a) illustrates a network modeling a texture image and (b) the degree of the vertices.

Figures 1(b) and (c) illustrate the mapping of the network onto a surface. Note that the $Z$ axis is the degree of the vertices. The dilation process of this surface is shown in Figures 1(d) and (e). Figure 2(a) shows the degree of vertices of a network modeling a texture image represented by an image. Figures 2(a)(c) show the dilatation process of the surface that represents this network with each point of the 3D surface dilated by a sphere of radius $r$. Notice that more collisions happen among the dilated points as we increase the value of radius $r$. These collisions are directly related to the geometry of the surface $S$ and determine the way the total influence volume $V_{S}(r)$ increases, according to the changes in the network topology. Thus, the pattern produced by the influence volume $V_{S}(r)$ for different radii can be used to describe the network topology and, consequently, the texture patterns.

\subsection{Feature Vector}

In this paper, we propose to use the influence volume $V_{S}(r)$ of the surface (that maps a network) as a feature vector. The influence volume provides a rich shape descriptor and it was used with success to discriminate texture in previous works $[17,4]$. Thus, the feature vector is composed of the concatenation of the influence volume $V_{S}(r)$ computed for different values of radius $r$ :

$$
\Psi_{t}^{d}=\left[V_{S}(1), V_{S}(\sqrt{2}), V_{S}(\sqrt{3}), \ldots, V_{S}\left(r_{\max }\right)\right],
$$

where $t$ is the value of threshold applied in the network, $d$ is the distance used in network modeling and $r_{\max }$ is the maximum radius.

Note that the feature vector $\Psi_{t}^{d}$ is built using all possible radius values from $r=1$ to $r_{\max }$. In order to analyze the behavior of the network topology from the successive transformation, we combine the feature vector $\Psi_{t}^{d}$ for different values of threshold $t$, starting from $t_{0}$ to $t_{f}$, to finally get the complete texture signature as follows:

$$
\Upsilon^{d}=\left[\Psi_{t_{0}}^{d}, \Psi_{t_{1}}^{d}, \ldots, \Psi_{t_{f}}^{d}\right]
$$

\section{Results and Discussion}

\subsection{Experimental setup}

The proposed signature is computed for each image. After that, the supervised classification is carried out by applying a Canonical Analysis [11] followed by a Linear Discriminant Analysis (LDA) [16]. This is a simple classifier that emphasizes the features extracted by the methods. We apply the Canonical Analysis here due to the presence of high correlation of the volume features [39]. Basically, the canonical analysis is a geometric transformation of the feature space to generate new uncorrelated features. Thus, $p$-canonical variables can be obtained from 

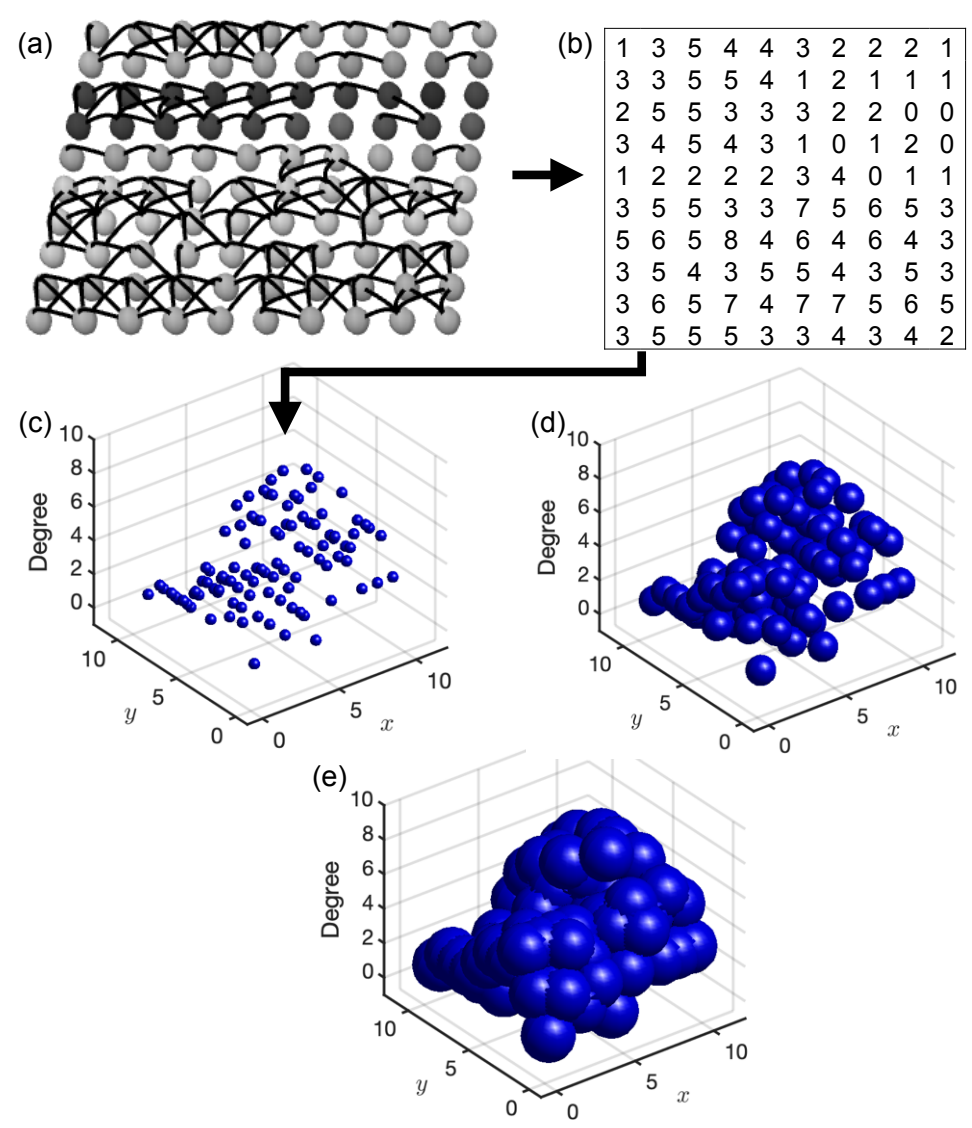

Fig. 1. Illustration of the proposed approach inspired by the Bouligand-Minkowski method for estimating the fractal dimension of a network. (a) Network modeling pixels. (b) Degree of the vertices. (c) 3D surface mapping the network in (a), converting each vertex into a $3 \mathrm{D}$ point. (d)-(e) Dilation process of the surface. 


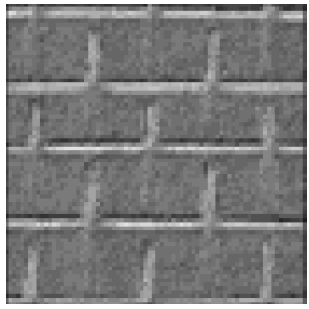

(a) Degree of vertices

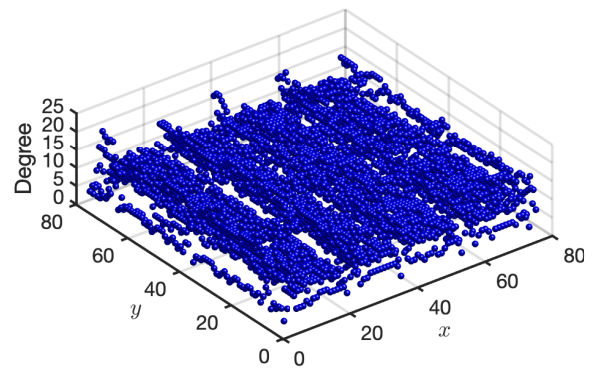

(c) $r=2$

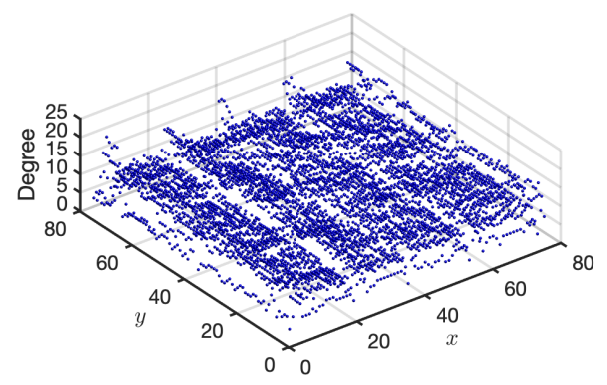

(b) $3 \mathrm{D}$ space

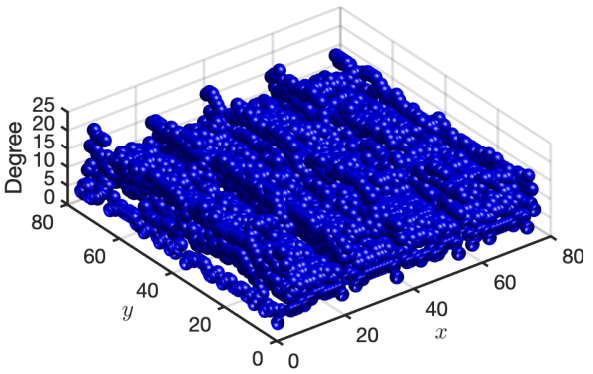

(d) $r=3$

Fig. 2. Dilation process for estimating the fractal dimension of the space $\times$ degree surface representing a texture. Degree of vertices (a) is mapped onto a 3D surface (b) by converting the Cartesian position and the degree into a point in the surface. Then, this surface is dilated, e.g. radius $r=2$ (c) and radius $r=3$ (d). 
the original features and the LDA supervised classification is accomplished by using the most significant $p$-variables [39]. To separate the training and testing sets we adopted the leave-one-out cross-validation scheme. Thus, one sample is used for testing and the remainder for training. This process is repeated for each sample of the dataset. The average accuracy of all tests is used as a performance measure.

To evaluate the methods the databases used as benchmark were:

- Brodatz [8]: just as in [7], this dataset is composed of 1776 texture images of $128 \times 128$ pixel size divided into 111 classes, 16 images per class.

- Outex [31]: the dataset used in this work is composed of 68 texture classes from TC_Outex_00013 with 20 samples each of $128 \times 128$ pixel size without overlapping. The dataset has a total of 1360 textures.

- USPTex [6]: this dataset is composed of 2292 samples divided into 191 classes, 12 images per class, and each image has $128 \times 128$ pixels size.

- Vistex [35]: the database Vision Texture has 54 images $512 \times 512$ which were split into 16 sub-images $128 \times 128$ pixel size without overlapping, totalizing 864 images.

We compare the accuracy of our proposed method to other descriptors proposed in the literature. For fair comparison purposes, all methods are compared using the LDA classifier and leave-one-out cross-validation scheme. The compared methods are: Grey-Level Co-occurrence Matrix (GLCM) [21], Gray Level Difference Matrix (GLDM) [26], Windowed Fourier transforms [3], Gabor Filters [30], Fractal [5], Fractal Fourier [14], Local Binary Patterns (LBP) [32], Local Binary Patterns Variance (LBPV) [20], Complete Local Binary Pattern (CLBP) [19], Local Phase Quantization (LPQ) [33], Local Configuration Pattern (LCP) [18], Local Frequency Descriptor (LFD) [28], Binarized Statistical Image Features (BSIF) [25] and Complex Network Texture Descriptors (CNTD) [7].

\subsection{Parameter Evaluation}

In this paper, the set of thresholds $T$ and distance for connection $d$ used are identical as the ones used in [7]. It was defined by an initial threshold $t_{0}=0.005$, an increment of $\Delta t=0.015$ and a final threshold $t_{f}=0.530$. The maximal distance $d$ for connection of the vertices was set up as $d=3$. Figure 3(a) shows the accuracy variation with respect to the number of the p-canonical variables used in the LDA classifier for different datasets and with a fixed value of maximal radius $r=3$. Note that, for all datasets, the accuracy increases at a first moment, achieves an optimal accuracy and then stabilizes. Such behavior is expected and is a good indicator for adjusting the dimension of the descriptor, since the high number of features damages the efficiency of the classifier [39]. From the behavior observed in Figure 3(a), we accomplish the remainder of the experiments using a total of $130 p$-canonical variables.

Figure 3(b) presents the accuracy variation for all datasets when the maximal value of radius $r_{\max }$ is ranged. The original feature vector before the canonical 
analysis has the size of 36, 144, 288 ,504, 792, 1116, 1512, 1944, 2484, 3060, $3672,4356,5112,5940,6804$ for the 15 respective integer values of $r_{\max }$ between 1 and 15 . We observe that, as we increase the maximal radius value the accuracy also increases. However, it begins to stabilize or decrease from the radius $r_{\max }=5$ and $r_{\max }=6$. Based on this behavior, we define the value of radius $r_{\max }=6$ as the maximum dilation radius. Therefore, we define the following feature vector $\Psi_{t}^{d}=\left[V_{S}(1), V_{S}(\sqrt{2}), V_{S}(\sqrt{3}), \ldots, V_{S}(\sqrt{36})\right]$.

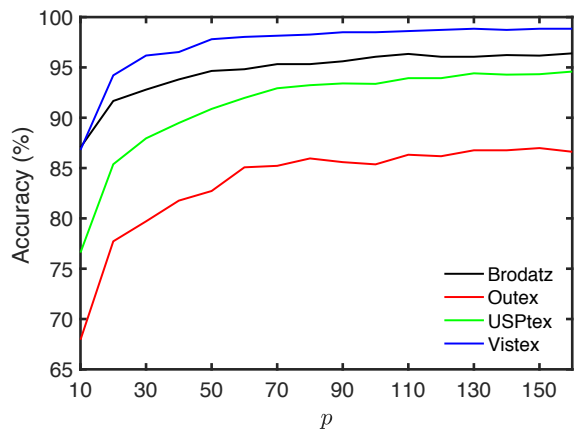

(a)

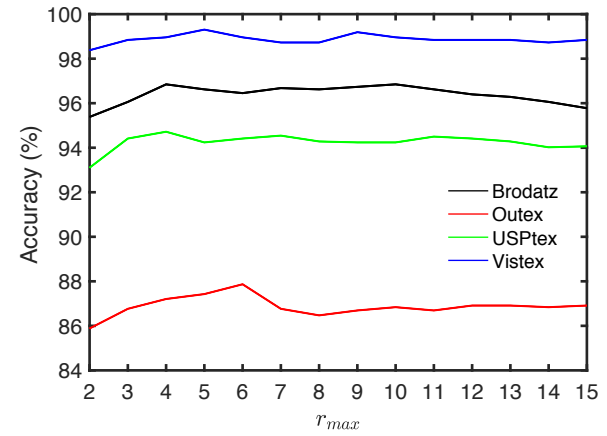

(b)

Fig. 3. Accuracy of our approach for different datasets. (a) Accuracy versus number of $p$-canonical variables. (b) Accuracy versus the maximal values of the radius.

\subsection{Comparison with other methods}

In this section, we performed comparisons with literature methods in order to evaluate the performance obtained by the proposed approach. In all experiments were used the LDA classifier with leave-one-out, except for CLBP descriptor (1-Nearest Neighborhood (1-NN) classifier with distance Chi-square), which followed the original paper. For our approach, it was adopted the parameter setup discussed in section 3.2.

The results obtained by all the texture methods in the four datasets are presented in Table 1. We can note that the proposed approach achieved the best results when compared to the other literature methods in the four datasets. In the Brodatz and Vistex datasets, the CLBP and CNDT methods obtained the second best results. On the other hand, the second best accuracy in the Outex and USPTex datasets was obtained by the GLDM and CNDT methods.

We can also verify in Table 1 that our method reached higher accuracy than the CNDT method. The CNDT method is also based on complex networks, and we use in our approach the same network modeling as them. The difference between the CNDT method and our approach is the measure (statistical measures based on the degree histogram) used to characterize the network. Therefore, this 
suggests that our approach obtained superior performance due to the fractal measure extracted from the network. Our interpretation is that our volumetric fractal descriptors can characterize more richly the network topology, considering the degree and spatial arrangement of the vertices, in contrast with the CNDT method that only used the degree frequency.

On the other hand, the proposed method also outperformed the Fractal method [5] which also uses the volumetric fractal descriptors to describe the image. However, this approach uses the Cartesian coordinates and the gray intensities to represent the surface. This shows that the proposed approach that combines fractal measures and complex networks improves the ability of discrimination when compared to both approaches isolated.

We can also compare our results with learned descriptors (e.g. using convolutional neural network $(\mathrm{CNN}))$. For comparison purposes, we considered the Outex and USPTex datasets, which are the most challenging. The results using the HardNet++ [2], InceptionV3 [40] and ResNet101 [23] methods for feature extraction were considered. On the USPTex dataset, our approach obtained the highest accuracies when compared to the InceptionV3 $(92.71 \%)$ and HardNet++ $(94.20 \%)$. On the other hand, the ResNet101 obtained an accuracy of $96.50 \%$. For the Outex dataset, the accuracies were $86.98 \%, 88.97 \%$ and $89.34 \%$ for the InceptionV3, ResNet101 and HardNet++, respectively. Our approach overcomes the CNN methods in some cases and in others it obtain close results. Thus, our method is still competitive due to its simplicity.

Table 1. Comparison of accuracies of different texture analysis methods in four texture databases.

\begin{tabular}{lccccc}
\hline Methods & Number of features Outex & USPTex & Brodatz & Vistex \\
\hline GLCM [21] & 24 & 80.73 & 83.63 & 90.43 & 92.24 \\
GLDM [26] & 60 & 86.76 & 91.92 & 94.43 & 97.11 \\
Gabor Filters [30] & 64 & 81.91 & 83.19 & 89.86 & 93.28 \\
Fourier [3] & 63 & 81.91 & 67.70 & 75.90 & 79.51 \\
Fractal [5] & 69 & 80.51 & 78.22 & 87.16 & 91.67 \\
Fractal Fourier [14] & 68 & 68.38 & 59.45 & 71.96 & 79.75 \\
LBP [32] & 256 & 81.10 & 85.42 & 93.64 & 97.92 \\
LBPV [20] & 555 & 75.66 & 55.13 & 86.26 & 88.65 \\
CLBP [19] & 648 & 85.80 & 91.13 & 95.32 & 98.03 \\
BSIF [25] & 256 & 77.43 & 77.48 & 91.44 & 88.66 \\
LCP [18] & 81 & 86.25 & 91.31 & 93.47 & 94.44 \\
LFD [28] & 276 & 82.57 & 83.59 & 90.99 & 94.68 \\
LPQ [33] & 256 & 79.41 & 85.29 & 92.51 & 92.48 \\
CNTD [7] & 108 & 86.76 & 91.71 & 95.27 & 98.03 \\
\hline Proposed approach & 130 & 87.86 & 94.41 & 96.45 & 98.96 \\
\hline
\end{tabular}




\section{Conclusion}

In this paper, we have proposed a new method based on fractal descriptors and complex network for texture analysis. The method extracts fractal measures from a network that models a texture image. For this, we have proposed to map the network into a 3D surface by converting the Cartesian coordinates and the vertex degree as a point in the surface.

We have demonstrated how the texture description can be improved by combining the fractal descriptors and complex networks, instead of using only the fractal descriptor or complex network approaches separated. Experiments on four datasets indicate that our method significantly improved the classification rate with regard to the original complex networks method and fractal descriptors method. The results also showed that our approach overcomes the other compared literature methods. As future work, we believe that different techniques to estimate the fractal dimension of the network can be investigated. In addition, new ways to obtain the feature vector can be studied, such as using different sets of radius values. Another future idea is to extend the proposed approach to boundary shapes analysis. The proposed idea also opens a promising research field for network characterization using fractal measures.

\section{Acknowledgments}

Lucas Ribas gratefully acknowledges the financial support grant \#2016/23763-8 and \#2019/03277-0, São Paulo Research Foundation (FAPESP). Odemir Bruno thanks the financial support of CNPq (Grant \#307797/2014-7) and FAPESP (Grant \#s 14/08026-1 and 16/18809-9).

\section{References}

1. Dynamic texture analysis with diffusion in networks. Digital Signal Processing 92, $109-126(2019)$

2. Anastasiya Mishchuk, Dmytro Mishkin, F.R.J.M.: Working hard to know your neighbor's margins: Local descriptor learning loss (Dec 2017)

3. Azencott, R., Wang, J.P., Younes, L.: Texture classification using windowed Fourier filters. IEEE Transactions on Pattern Analysis and Machine Intelligence 19(2), 148-153 (1997)

4. Backes, A.R., Bruno, O.M.: Plant leaf identification using color and multi-scale fractal dimension. In: International Conference on Image and Signal Processing. pp. 463-470 (2010)

5. Backes, A.R., Casanova, D., Bruno, O.M.: Plant leaf identification based on volumetric fractal dimension. International Journal of Pattern Recognition and Artificial Intelligence 23(06), 1145-1160 (2009)

6. Backes, A.R., Casanova, D., Bruno, O.M.: Color texture analysis based on fractal descriptors. Pattern Recognition 45(5), 1984-1992 (2012)

7. Backes, A.R., Casanova, D., Bruno, O.M.: Texture analysis and classification: A complex network-based approach. Information Sciences 219, 168-180 (2013) 
8. Brodatz, P.: Textures: A photographic album for artists and designers. Dover Publications, New York (1966)

9. Bruno, O.M., de Oliveira Plotze, R., Falvo, M., de Castro, M.: Fractal dimension applied to plant identification. Information Sciences 178(12), 2722 - 2733 (2008)

10. Chalumeau, T., Costa, L.d., Laligant, O., Meriaudeau, F.: Texture discrimination using hierarchical complex networks. In: Damiani, E., Yatongnon, K., Schelkens, P., Dipanda, A., Legrand, L., Chbeir, R. (eds.) Signal Processing for Image Enhancement and Multimedia Processing, Multimedia Systems and Applications Series, vol. 31, pp. 95-102. Springer US (2008)

11. Correa, D.C., Saito, J.H., da F Costa, L.: Musical genres: beating to the rhythms of different drums. New Journal of Physics 12(5), 053030 (may 2010)

12. Costa, L.d.F., Rodrigues, F.A., Travieso, G., Villas Boas, P.R.: Characterization of complex networks: A survey of measurements. Advances in Physics 56(1), 167-242 (2007)

13. Fabbri, R., Costa, L.D.F., Torelli, J.C., Bruno, O.M.: 2d euclidean distance transform algorithms: A comparative survey. ACM Computing Surveys (CSUR) 40(1), $2(2008)$

14. Florindo, J.B., Bruno, O.M.: Fractal descriptors based on Fourier spectrum applied to texture analysis. Physica A: statistical Mechanics and its Applications 391(20), 4909-4922 (2012)

15. Florindo, J.B., Casanova, D., Bruno, O.M.: Fractal measures of complex networks applied to texture analysis. In: Journal of Physics: Conference Series. vol. 410, p. 012091. IOP Publishing (2013)

16. Fukunaga, K.: Introduction to Statistical Pattern Recognition. Academic Press, 2nd edn. (1990)

17. Goncalves, W.N., Machado, B.B., Bruno, O.M.: Texture descriptor combining fractal dimension and artificial crawlers. PHYSICA A-STATISTICAL MECHANICS AND ITS APPLICATIONS 395, 358-370 (2014)

18. Guo, Y., Zhao, G., Pietikäinen, M.: Texture classification using a linear configuration model based descriptor. In: BMVC. pp. 1-10. Citeseer (2011)

19. Guo, Z., Zhang, L., Zhang, D.: A completed modeling of local binary pattern operator for texture classification. IEEE Transactions on Image Processing 19(6), 1657-1663 (2010)

20. Guo, Z., Zhang, L., Zhang, D.: Rotation invariant texture classification using lbp variance (lbpv) with global matching. Pattern recognition 43(3), 706-719 (2010)

21. Haralick, R.M.: Statistical and structural approaches to texture. Proceedings of the IEEE 67(5), 786-804 (1979)

22. Haralick, R.M., Shanmugam, K., Dinstein, I.H.: Textural features for image classification. Systems, Man and Cybernetics, IEEE Transactions on (6), 610-621 (1973)

23. He, K., Zhang, X., Ren, S., Sun, J.: Deep residual learning for image recognition. In: Proceedings of the IEEE conference on computer vision and pattern recognition. pp. 770-778 (2016)

24. Jain, A.K., Farrokhnia, F.: Unsupervised texture segmentation using Gabor filters. In: Systems, Man and Cybernetics, 1990. Conference Proceedings., IEEE International Conference on. pp. 14-19. IEEE (1990)

25. Kannala, J., Rahtu, E.: Bsif: Binarized statistical image features. In: Pattern Recognition (ICPR), 2012 21st International Conference on. pp. 1363-1366. IEEE (2012)

26. Kim, J.K., Park, H.W.: Statistical textural features for detection of microcalcifications in digitized mammograms. IEEE transactions on medical imaging 18(3), 231-238 (1999) 
27. Lam, W.K., Li, C.K.: Rotated texture classification by improved iterative morphological decomposition. IEE Proceedings-Vision, Image and Signal Processing 144(3), 171-179 (1997)

28. Maani, R., Kalra, S., Yang, Y.H.: Noise robust rotation invariant features for texture classification. Pattern Recognition 46(8), 2103-2116 (2013)

29. Malamas, E.N., Petrakis, E.G., Zervakis, M., Petit, L., Legat, J.D.: A survey on industrial vision systems, applications and tools. Image and vision computing 21(2), 171-188 (2003)

30. Manjunath, B.S., Ma, W.Y.: Texture features for browsing and retrieval of image data. IEEE Transactions on pattern analysis and machine intelligence 18(8), 837$842(1996)$

31. Ojala, T., Mäenpää, T., Pietikäinen, M., Viertola, J., Kyllönen, J., Huovinen, S.: Outex: New framework for empirical evaluation of texture analysis algorithms (2002)

32. Ojala, T., Pietikainen, M., Maenpaa, T.: Multiresolution gray-scale and rotation invariant texture classification with local binary patterns. IEEE Transactions on pattern analysis and machine intelligence 24(7), 971-987 (2002)

33. Ojansivu, V., Heikkilä, J.: Blur insensitive texture classification using local phase quantization. In: International conference on image and signal processing. pp. 236243. Springer (2008)

34. Panjwani, D.K., Healey, G.: Markov random field models for unsupervised segmentation of textured color images. IEEE Transactions on pattern analysis and machine intelligence 17(10), 939-954 (1995)

35. Picard, R., Graczyk, C., Mann, S., Wachman, J., Picard, L., Campbell, L.: Vision texture database. Media Laboratory, MIT, Cambridge, Massachusetts (1995)

36. Ribas, L.C., Junior, J.J., Scabini, L.F., Bruno, O.M.: Fusion of complex networks and randomized neural networks for texture analysis. arXiv preprint arXiv:1806.09170 (2018)

37. Ribas, L.C., Gonçalves, D.N., Oruê, J.P.M., Gonçalves, W.N.: Fractal dimension of maximum response filters applied to texture analysis. Pattern Recognition Letters 65, 116-123 (2015)

38. Ribas, L.C., Neiva, M.B., Bruno, O.M.: Distance transform network for shape analysis. Information Sciences 470, 28 - 42 (2019)

39. da S. Oliveira, M.W., Casanova, D., Florindo, J.B., Bruno, O.M.: Enhancing fractal descriptors on images by combining boundary and interior of minkowski dilation. Physica A: Statistical Mechanics and its Applications 416, 41 - 48 (2014)

40. Szegedy, C., Vanhoucke, V., Ioffe, S., Shlens, J., Wojna, Z.: Rethinking the inception architecture for computer vision. In: Proceedings of the IEEE conference on computer vision and pattern recognition. pp. 2818-2826 (2016)

41. de Ves, E., Acevedo, D., Ruedin, A., Benavent, X.: A statistical model for magnitudes and angles of wavelet frame coefficients and its application to texture retrieval. Pattern Recognition 47(9), 2925 - 2939 (2014)

42. Wenk, H.R.: Preferred Orientation in Deformed Metal and Rocks: An Introduction to Modern Texture Analysis. Elsevier (2013)

43. Zimer, A.M., Rios, E.C., Mendes, P.d.C.D., Gonçalves, W.N., Bruno, O.M., Pereira, E.C., Mascaro, L.H.: Investigation of aisi 1040 steel corrosion in h2s solution containing chloride ions by digital image processing coupled with electrochemical techniques. Corrosion Science 53(10), 3193-3201 (2011) 